\title{
Plasma-wall interaction: how atomic processes influence the performance of fusion plasmas
}

\author{
Ralf Schneider \\ Max-Planck Institute for Plasmaphysics, EURATOM Association, Wendelsteinstr. 1, D-17491 \\ Greifswald, Germany
}

\begin{abstract}
Plasma edge physics is one of the major challenges in fusion plasmas. The need for power and particle exhaust for any reactor inspired a lot of theoretical and experimental work. Understanding this physics requires a multi-scale ansatz bringing together also several physics and numerical models.

The plasma edge of fusion experiments is characterized by atomic and molecular processes. Hydrogenic ions and neutrals hit material walls with energies from several $\mathrm{eV}$ up to $1000 \mathrm{~s}$ of eV. They saturate the wall materials and due to physical or chemical processes neutrals are released from the wall, both atomic and molecular. They determine via interaction with the plasma strongly its properties. These processes can be beneficial for a fusion experiment by using radiation losses to minimize the power load problem of target plates, but also can create severe problems if the dilution of the plasma gets too large or condensation radiation instabilities can be created.

A complete physics model for the plasma-wall interaction processes alone is already rather challenging (and still missing): it requires e.g. inclusion of collision cascades, chemical formation of molecules, diffusion in strongly 3D systems. A full description needs a multi-scale model combining quite different numerical techniques like molecular dynamics, binary collisions, kinetic Monte Carlo and mixed conduction/convection equations in strongly anisotropic systems.
\end{abstract}

Keywords: Fusion plasmas, plasma-wall interaction, neutrals, impurities

PACS: 52.20.-j, 52.25.Jm, 52.25.Vy, 52.40.Hf, 52.55.Rk

\section{INTRODUCTION}

Fusion is one of the options for overcoming the energy problems in this century. Sufficient good energy confinement to achieve ignition is obtained in magnetic fusion by the creation of a magnetic cage of nested flux surfaces using the enormous anisotropy between transport along fieldlines and perpendicular to it. The compression of helium and the helium pumping is important, because one has to avoid too much helium ash which would extinguish the burning plasma in a reactor. A global OD model for burning D-T plasmas addresses the effect of impurities on ignition conditions [1,2]. Depending on the assumed concentration of additional impurities, one only gets solutions if the ratio of the global $\alpha$-particle confinement time and the energy confinement time is less than 10-15. Otherwise, the helium ash just extinguishes the burning plasma.

The scrape-off layer region gets very important in any fusion reactor, because it is directly related to the requirements for power and particle exhaust compatible with the core confinement. One major problem is that the thickness of the layer where the heat flux from the core is transported onto walls is rather small (typically about $1 \mathrm{~cm}$, which is the size of the poloidally projected ion gyro-radius) due to the very strong parallel transport, especially for the required enhanced confinement modes necessary 
for ignition. This results in severe engineering problems due to exceedingly large power loads on the walls, illustrated here for ITER and motivating the need for a detailed understanding of the scrape-off layer physics.

In ITER the amount of power carried by the $\alpha$-particles $\mathrm{P}_{\alpha}$ has to be exhausted in a controlled way. Doing a simple estimate for the ITER target-plate power load $q_{\perp, t p}$, one gets

$$
q_{\perp, t p}=\frac{P_{\alpha}}{2 \pi \cdot R_{X-p o i n t} \cdot 2 \cdot \Delta_{e}},
$$

where $\Delta_{e}$ is the energy decay length at the target-plate and $R_{X \text {-point }}$ is the major radius at the $X$-point. For this estimate one assumes the deposition of the power in a strip of $\Delta_{e}$ on two divertor plates on a ring with length $2 \pi R_{X-p o i n t}$.

This gives for the planned ITER [3]

$$
q_{\perp, t p}=\frac{300 \mathrm{MW}}{4 \pi \cdot 7 \mathrm{~m} \cdot 0.02 \mathrm{~m}}=175 \mathrm{MW} / \mathrm{m}^{2} .
$$

Introducing further reduction factors due to bulk radiation losses (0.8) and poloidal tilting of target-plates $(0.5-0.25)$ one still get values of about $q_{\perp, t p}=35 \mathrm{MW} / \mathrm{m}^{2}$. The reduction factor of $(0.5-0.25)$ by tilting of the target-plates is not as large as expected from flux expansion factors between midplane and target-plates which can easily be 0.1 and lower, because the total surface area does not change as much due to the fact that the intersection angles do not change so strongly. They even increase again if the tilting is very strong, because then the distance to the X-point gets larger in which vicinity the angles are very small.

Realistic values for steady-state operation are below about $5 \mathrm{MW} / \mathrm{m}^{2}$. This means that additional losses are required to spread the target power load over a wider area. It is important to note here that the final concept has to be compatible then also with the necessity of particle exhaust (helium ash removal) and core confinement.

A divertor is produced by additional coils creating a separatrix intersecting in the $X$ point. Here, the basic idea is to move the interaction zone away from the core to so-called divertor plates. It is hoped to get a better impurity control due to very good divertor retention and to achieve a reduction of the heat flux to target-plates due to radiation losses.

\section{ELEMENTARY PROCESSES IN THE DIVERTOR}

To lower the maximum power load and to broaden the energy deposition profile on the target-plates, one has to use loss channels which are not constricting the energy flow to follow magnetic field-lines. The ideal scenario would be one where these energy losses would be created without additional impurities. For hydrogen three energy exchange channels - charge exchange, atomic radiation and volume recombination - are in principle available (see Fig. 1).

Charge exchange losses. Charge exchange (CX) enery conversions are already used experimentally with great success to convert high energy fluxes of charged particle 


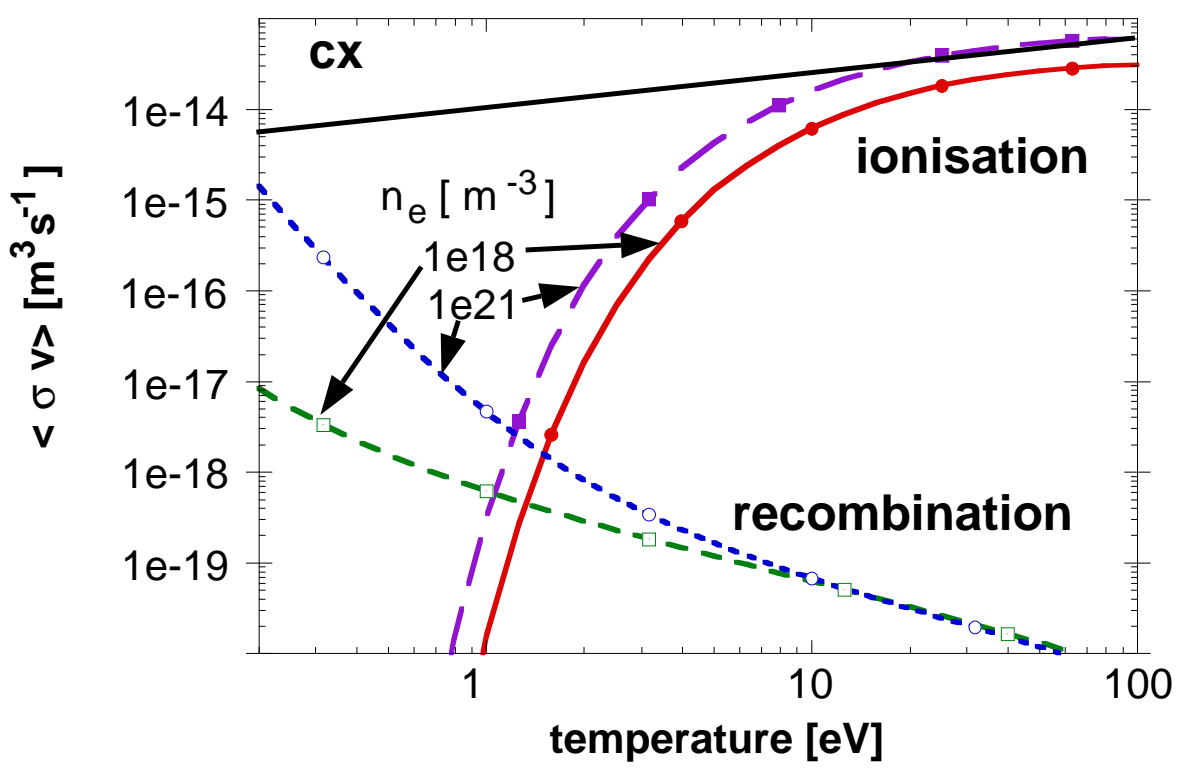

FIGURE 1. Cross sections for charge exchange (CX)), ionization and recombination as a function of electron temperature for hydrogen.

beams into neutrals through neutralizer sections in neutral injector beam lines. In divertors it requires, however, that the plasma be sufficiently thin, so that neutrals can interpenetrate it. For ITER parameters one gets losses of nearly 1 at $660 \mathrm{eV}$, but only 0.06 at $100 \mathrm{eV}$ and 0.002 at $10 \mathrm{eV}$. That means, that $\mathrm{CX}$ energy losses are only effective at high temperatures. Moreover, CX neutrals from the hot regions pose erosion problems in the main chamber and the transition region from the divertor to the main chamber (baffles, etc.).

Neutral hydrogen radiation. For neutral hydrogen atoms at electron temperatures above about $8 \mathrm{eV}$, the ratio of an enhanced 'effective' electron ionisation energy loss to the 'true' ionisation energy loss, which is again deposited along field-lines at the targetplate (without volume recombination) is around 2-3.

Only the radiated fraction, which arises during the stepwise ionisation through many excited states of the atom, constitutes a true volumetric loss. Below about $8 \mathrm{eV}$ the radiated power associated with ionisation processes increases significantly. The characteristics of the parallel electron heat conduction $\left(q_{\|} \sim T^{5 / 2} \nabla T \sim T^{7 / 2}\right)$ imply, however, that the volume over which the temperature can be below a certain value at a certain heat flux density is proportional to $T^{7 / 2}$ along field-lines, and hence dramatically decreases with temperature. Therefore, the low temperature region is very small and cannot contribute to the integral radiation loss.

Volume recombination. For plasma temperatures below about $2 \mathrm{eV}$, volume recombination processes become important. Recombination of an electron and an ion into a neutral atom needs a second body to account for energy and momentum conservation during the process. This is possible in two ways: in the process of radiative recombi- 
nation a photon takes care of energy and momentum conservation. The second process is three-body recombination where an additional electron ('spectator electron') is necessary. The recombination rate for this process gives the strong rise below about $2 \mathrm{eV}$ (especially for high densities above $10^{20} \mathrm{~m}^{-3}$ ). The second process is mainly responsible for the strong effects of volume recombination in the divertor affecting the whole character of the plasma state. Both processes proceed in a ladder-like way through the excited levels of the atoms until the final ground state is reached. Hence hydrogen line radiation is involved with a specific spectral distribution, which is important for diagnosing the onset of strong volume recombination spectroscopically.

The effective electron cooling rate for the recombination rates shows that the absolute direct energy loss for electrons by volume recombination is usually small and can be even a heating term for high densities and low temperatures. That means that the process of volume recombination has the tendency to stabilise the temperature and avoid further cooling. From what was said above it follows that the recombination zone is always located "below" the ionisation zone (i.e. between ionisation zone and plate).

Summarising this discussion of energy losses from hydrogen, it is obvious that for ITER additional radiation losses from impurities are necessary [8].

\section{Divertor geometry effects}

The target-plate geometry strongly influences the plasma profiles by controlling the neutral recycling pattern, which in turn has a strong effect on the symmetry and stability of the divertor plasma and finally on the whole edge region. The design of the new divertor configuration called Lyra for ASDEX Upgrade (see Fig. 2) is a good example of this optimisation strategy.
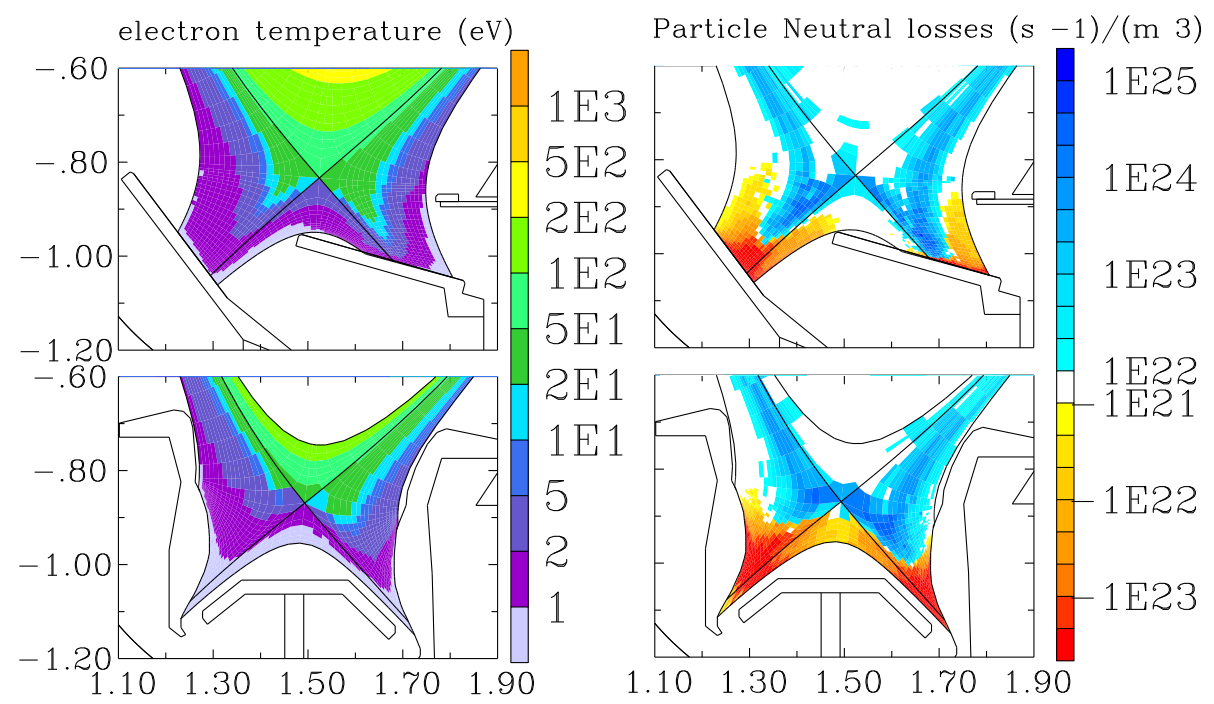

FIGURE 2. Contours of the temperatures and neutral sources as calculated with B2-Eirene. The blue sources are ionsisation sources, the red sources are recombination sinks for the plasma.

For Divertor-I, the outer divertor plate reflects the neutrals away from the energycarrying zone close to the separatrix and thus blocks indirectly the detachment (see 
Fig. 2). Therefore, Divertor-I detaches strongly asymmetrically (preferentially on the inner divertor)as can be seen in the temperature and neutral source distributions. Nevertheless, the power load on the outer target-plate is reduced by the large flux expansion close to the $X$-point. The Lyra has, for the same density conditions, the lowest power loads, because it reflects the neutrals towards the high energy zone at the separatrix and is strongly tilted. Therefore, concerning the operational safety of the machine, it offers the best configuration to start with, allowing for scenarios without external impurities and the largest heating power.

\section{Effect of vibrationally excited molecules}

A mechanism, possibly also important in the divertor, is recombination through vibrationally excited molecules. Two possible reaction paths exist. The first one is the creation of negative ions (e+ $\mathrm{H}_{2}(v) \rightarrow \mathrm{H}_{2}^{-}$), followed by dissociation $\left(\mathrm{H}_{2}^{-} \rightarrow \mathrm{H}+\mathrm{H}^{-}\right.$and $\left.\mathrm{H}^{+}+\mathrm{H}^{-} \rightarrow \mathrm{H}+\mathrm{H}^{*}\right)$. The second channel is ion conversion $\left(\mathrm{H}^{+}+\mathrm{H}_{2}(v) \rightarrow \mathrm{H}+\mathrm{H}_{2}^{+}\right)$and dissociation $\left(\mathrm{e}+\mathrm{H}_{2}^{+} \rightarrow \mathrm{H}+\mathrm{H}^{*}\right)$.

The rate coefficients of these two processes lead to a large increase of the total recombination rate coefficient (including also three-body and radiative recombination) by at least one order of magnitude for temperatures below about $5 \mathrm{eV}$. However, the rate coefficients for ionisation and dissociation increase also, reducing thus the mean free path of the molecules. The recombination source depends on rate coefficient and electron density, but also on the molecular density. Therefore, a large recombination rate coefficient does not necessarily lead to large recombination effects (sources). A detailed discussion of this can be found in $[4,5]$.

Introducing the different vibrational levels as separate species in the neutral MonteCarlo-Code Eirene and doing a self-consistent B2-Eirene calculation this recombination channel is only important in a rather thin surface layer close to the plasma boundary, because the neutral molecules do not penetrate as deep as for the case without taking into account the vibrational excitation. The integral contribution to the total recombination rate is also a minor correction.

\section{IMPURITY PHYSICS}

After the discussion of the clean plasma, the SOL description must be completed by including the effect of impurities. Fusion plasmas always contain impurities, be it $\mathrm{He}$ fusion ash, radiative gases injected $\left(\mathrm{N}_{2}, \mathrm{Ar}, \mathrm{Ne}, \mathrm{Kr}\right)$, or wall material sputtered by the plasma $(\mathrm{C}, \mathrm{W}, \mathrm{Fe}, \mathrm{Mo}, \mathrm{Ni}, \ldots)$. Therefore, for the understanding of impurity physics in the SOL, one needs a rather complex combination of different aspects. The impurity production process has to be understood, then the effects of impurities in terms of radiation losses have to be included, and finally the impurity transport is necessary. 


\section{Impurity production}

The radiation losses by impurities are necessary for relaxing the heat exhaust problem by spreading the heat deposition onto a larger surface by radiation. However, radiation losses in the main chamber can be a problem, because a minimum amount of power is necessary in order to sustain sufficient confinement (H-mode threshold) and to sustain ignition one has to avoid too large power losses. Therefore, a scenario is needed where impurity losses (power as well as position) can be (feedback) controlled. Intrinsic impurities $(\mathrm{C}, \mathrm{W}, \mathrm{Be}, \ldots)$ are produced through erosion (ions, $\mathrm{CX}$ neutrals, hot spots, arcs) and melting (disruptions, very large ELMs). The physical sputtering process is a binary collision between surface or bulk atoms and impinging ions (after acceleration in the sheath) or neutrals [6]. Due to the binary collisions, one gets a threshold energy for the impinging particle below which the sputtering yield drops very fast to practically zero. This threshold is therefore higher for heavier bulk particles.

Seeded impurities for controlled impurity losses have to be recycling impurities (noble gases, e.g. Ne, Ar, Kr), because non-recycling impurities cannot be pumped for feedback control but stick to the walls. For the choice of target (and main chamber) materials, one has several candidates representing either low $Z$ (e.g. Be, C) or high $Z$ (e.g. Mo, W) materials, which both have different advantages and disadvantages. Carbon has a very high thermal conductivity, high melting point and low vapour pressure, but is subject to chemical erosion as it readily combines chemically with hydrogen isotopes, leading in particular to tritium wall inventory problems in a reactor environment. Concerning the sputtering by ions (after acceleration in the sheath) and high energy CX neutrals, tungsten has much lower effective sputtering yields than beryllium or carbon. This is due to the advantageous ratio of ionisation-length to ion gyro-radius [7] leading to a high probability of prompt redeposition within the first ion gyration of the tungsten ion after ionisation of the sputtered atom. However, only a very small fraction of $\mathrm{W}$ in the main plasma $\left(2 \cdot 10^{-5}\right)$ would be enough to quench ignition. Molybdenum raises concerns due to its activation under neutron bombardment, leading to the creation of relatively longlived radioactive isotopes within the wall material. Beryllium has the problem of a low melting point and high toxicity, but excellent vacuum gettering properties.

The radiation losses for the low $Z$ elements are dominated for temperatures below $100 \mathrm{eV}$ by line radiation losses and above several keV by bremsstrahlung. Due to their radiation characteristics, low $Z$ elements will contribute more to SOL and divertor radiation (temperatures below $100 \mathrm{eV}$ ), whereas with higher $Z$ more and more radiation will move to the confinement region of closed field lines (Fig. 3). For tungsten, the radiation losses are three orders of magnitude larger than for carbon or beryllium. Therefore, one needs nearly perfect divertor retention for tungsten in contrast to carbon. This gives a very strict limit for main chamber concentration $\left(10^{-5}\right)$ for ITER, and up to now it is not clear whether this is achievable.

For carbon one has a different problem to overcome: even at low plasma temperatures, where physical sputtering switches off, sputtering of carbon is still continuing. The reason is chemical sputtering producing methane and other hydrocarbons, which finally break up into carbon ions [9]. This process is a surface effect and has a strong temperature dependence with a maximum yield at about $600 \mathrm{~K}$, characterised by the change of the carbon bonds at a surface from an $s p^{3}$ to $s p^{2}$ type. This process gives large erosion 


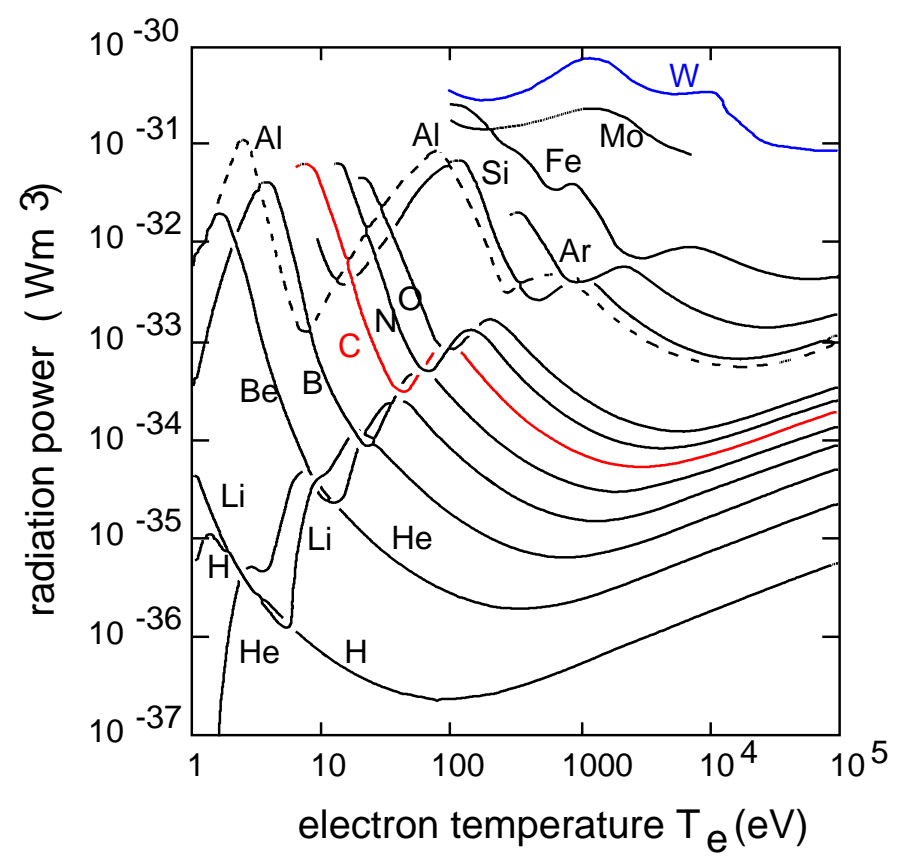

FIGURE 3. Radiation power $\frac{P_{\text {rad }}}{\operatorname{Vol} \cdot n_{e} \cdot n_{i m p}}$ as a function of electron temperature.

rates for ITER, due to the very large incoming particle fluxes, although there exists a favourable flux dependence of the chemical sputtering yield [10]. Some experimental data also points to a total fluence dependence, but are still unexplained [11]. Possibilities to overcome this problem for carbon are proposed and tested by titanium doping for suppression of the chemical erosion. Other experiments suggest that nitrogen injection may also contribute to reducing chemical erosion [12], likely by a catalytic 'poisoning' of the active carbon sites available.

The hydrocarbons produced by chemical sputtering also tend to form co-deposited layers far away from the plasma like in pump ducts. This results from additional transport through neutrals and/or low temperature plasmas in the periphery. No exact geometrical configuration can be given for these a-C:H films and the material must be characterized in another way. Three of the most important characteristics of an a-C:H are the hydrogen content, $\mathrm{sp}^{2} / \mathrm{sp}^{3}$ bonding ratio and the density of the sample. Low density and high hydrogen concentration $(\sim 50-70$ at.$\%)$ a-C:H films are generally referred to as polymer or soft a-C:H, whereas, high density, high $\mathrm{sp}^{3}$ and low hydrogen concentration (up to 30 at.\%) hydrocarbon films are usually reffered to as diamondlike carbon (DLC) films or hard $\mathrm{a}-\mathrm{C}: \mathrm{H}[15]$. These layers pose a severe safety problem for any reactor because they trap a huge amount of tritium. Therefore, understanding of such processes and plasmas is critical for fusion reactor design and will strongly influence the choice of the wall material. 


\section{Radiation losses}

ITER needs additional radiation losses to overcome the power exhaust problem as discussed before. However, there exists a rigid $Z_{\text {eff }}$ limit. Only $\Delta Z_{\text {eff }}=0.2$ is available for seed impurities, otherwise due to too strong bremsstrahlung losses it is not possible to sustain ignition [13]. This results in a fatal fraction $f_{Z}$ for $\mathrm{Be}(0.14), \mathrm{C}(0.07), \mathrm{Ne}(0.025)$, $\operatorname{Ar}(0.0054)$. Using now the planned ITER parameter $n_{e} \approx 1 \cdot 10^{20} \mathrm{~m}^{-3}$, one can estimate the necessary radiation losses on closed fieldlines, which would result in too low power fluxes at the separatrix incompatible with a good confinement. This needs a minimum power flux crossing the separatrix, because $\mathrm{H}$-mode power threshold predictions for ITER are between 70-300 MW.

On open fieldlines one can use different radiators (e.g. neon, carbon and hydrogen, which radiate most strongly at lower and lower temperatures). Using several losses in series (different radiators, like neon, carbon and hydrogen) one gets larger losses, because after each loss the normalised input power gets smaller and this moves the operational point to larger relative losses. Due to the fact that a reduction in the heat flux density increases the spatial extent of the region between two fixed isotherms one gets enhanced total power radiated away by a given impurity at given plasma pressure and impurity concentration. Using this synergy for radiation losses in series e.g. radiation from neutral hydrogen atoms can ultimately become significant (close to the target plate), once the major fraction of the total heating power has been radiated via other upstream loss channels.

Also,for power removal in ITER. one needs enhancements of the radiation loss function beyond coronal equilibrium rates. The main reasons for such non-equilibrium situations are impurity transport and charge-exchange effects. The first (and most important) is the effect of transport, which produces a lag of the actual ionisation degree behind its equilibrium value. This is especially important for the limited residence time of impurities close to the plate, where fast recycling shifts the ionisation balance toward lower charge states. The second effect is $\mathrm{CX}$ recombination in the presence of neutral hydrogen $\left(\mathrm{H}^{0}+\mathrm{A}^{i+} \Rightarrow \mathrm{H}^{+}+\left(\mathrm{A}^{(i-1)+}\right)^{*}\right)$, which also shifts the ionisation balance toward lower charge states.

Using multi-fluid SOL transport codes like B2-Eirene to account for these effects, it is demonstrated that these non-equilibrium effects are strong enough that one gets enough radiation losses for sufficient power exhaust for ITER [13].

\section{MARFEs}

Another important phenomena is the occurrence of a radiation instability, a so-called MARFE (Multifaceted Asymmetric Radiation From the Edge). This radiation instability build up if one gets higher radiation losses at lower temperature. For the instability, the temperature dependence of the total sum of radiation losses (minus heating power) is important [14]. The radiation function of carbon exhibits (depending on the local heating power), for electron temperatures below about $50 \mathrm{eV}$, an unstable branch, leading to the formation of a condensation instability at low temperatures. This results in a 
transition from low radiation level (concentrated in the divertor) to rather large radiation levels (concentrated close to the $X$-point). The MARFE is then characterised by a low temperature region close to the $X$-point on closed field lines. Here, the assumption of pressure constancy along field-lines is very well fulfilled, resulting in quite large densities and therefore quite large radiation losses compared to midplane conditions. Due to the relatively low temperatures (below about $50 \mathrm{eV}$ ), the counteracting parallel heat conduction, which would try to keep the temperature up at the $X$-point to midplane values, decreases strongly with temperature and thus facilitates MARFE formation. The location can also be understood, because the cross-field heat source is minimal close to the $X$-point for a single null case due to the large expansion of flux surfaces in its vicinity. The $X$-point in a single null is also characterised by a very small effective heat conduction coefficient due to the shallow pitch angle there, creating strong temperature gradients poloidally. In other terms, due to the long connection length in this region the radiation has a tendency to focus here.

Comparing the radiation functions of the different elements it is obvious that one has the possibility to custom-tailor the total radiation function by combining different impurities, like $\mathrm{C}$ plus $\mathrm{Ne}$, and by this reduce the tendency for instability. In contrast to the low- $Z$ elements, medium or high- $Z$ do not have this pronounced instability branch in the radiation loss function. Therefore, using e.g. neon as a radiator can reduce the instability formation and allow higher radiation losses before a MARFE is formed. This radiation instability cools the plasma edge which can lead to growth of magnetic islands and finally to a disruption, if the current profile becomes unstable.

\section{PLASMA EDGE MODELING}

The basic problem of plasma edge physics is the large range of length (see Fig. 4) and corresponding time scales.

Plasma wall interaction effects introduce microscopic length scales (like the typical interaction distance of about $1 \mathrm{~nm}$ between atoms and molecules) and very short time scales (fast momentum transfer processes determining the collision processes occur in $10^{-12} \mathrm{~s}$ ). These processes are important for material changes in plasma wall facing materials and therefore also for the release of impurities into the plasma (e.g. physical or chemical sputtering). They are studied either by molecular dynamics (MD) or by a simplified binary collision model. The latter strongly reduces the computational effort, though at the price of drastically simplifying the physics. In addition, diffusion in such materials introduces length scales spanning from microns (size of the granules) up to centimeters (size of the tiles). These effects (e.g. diffusion in amorphous materials) are analyzed with Monte Carlo methods (kinetic Monte Carlo with input from molecular dynamics or experiment).

The plasma description again has different levels of complexity. A full kinetic description (including ions, electrons, neutrals and their collisions) is possible for some low temperature plasmas (e.g. electron cyclotron resonance heated methane plasmas) and for qualitative studies of edge plasma effects in fusion edge plasmas. Here, the limitations are given by the fact that the Debye length and the plasma frequency have to be resolved. 


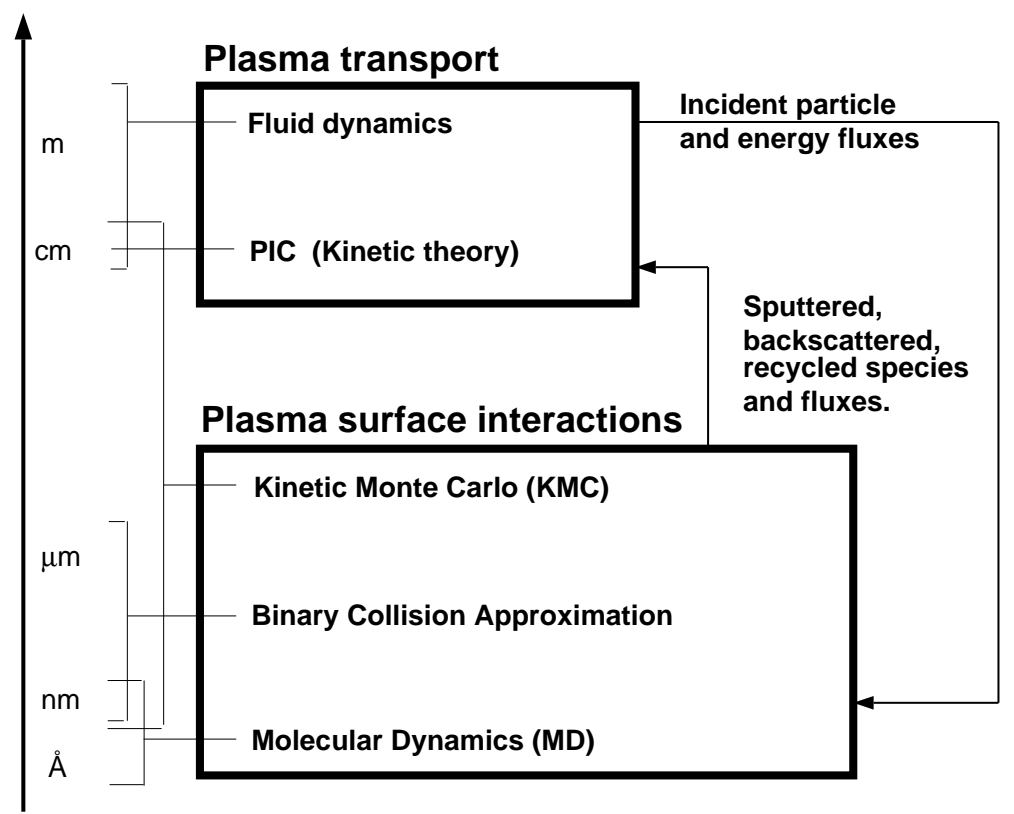

FIGURE 4. The different length scales and methods used for plasma edge modelling.

For the study of the physics of the edge of magnetically confined plasmas (2D tokamaks, tokamaks with ergodic perturbations, 3D stellarators) fluid codes are used for understanding the complex physics in such devices. Depending on the geometrical complexity (2D tokamaks, 3D stellarators) and on the additional effect of ergodicity, different numerical methods (finite volume, finite difference and Monte Carlo methods) are used.

The plasma surface interactions influence the plasma transport through sputtered, back scattered and recycled particles and fluxes. On the other hand, the incident particle and energy fluxes determine the plasma surface interaction.

\section{ACKNOWLEDGMENTS}

Ralf Schneider acknowledges funding of the work by the Initiative and Networking Fund of the Helmholtz Association.

\section{REFERENCES}

1. D. Reiter and G.H. Wolf and H. Kever, Nuclear Fusion 30, 2141-2155 (1990).

2. R. Behrisch and V. Prozesky, Nuclear Fusion 30, 2166-2169 (1990).

3. ITER physics base editors, ITER physics expert groups, JCT, Nuclear Fusion 39, 2137-2638 (1999).

4. Fantz, U. and Behringer, K. and Gafert, J. and Coster, D.P. and ASDEX Upgrade Team, Journal of Nuclear Materials 266-269, 490-494 (1999).

5. Fantz, U. and Reiter, D. and Heger, B. and Coster, D.P., Journal of Nuclear Materials 290-293, 367373 (2001). 
6. Eckstein, W., "Computer Simulation of Ion-Solid Interactions," in Springer Series in Materials Science, Vol.10, Springer, Berlin, 1991.

7. Naujoks, D. and Roth, J. and Krieger, K. and Lieder, G. and Laux, M., Journal of Nuclear Materials 210, 43-50 (1994).

8. Janeschitz, G. and Borrass, K. and Federici, G. and Igitkhanov, Y. and Kukushkin, A. and Pacher, H. D. and Pacher, G. W. and Sugihara, M., Journal of Nuclear Materials 220-222, 73-88 (1995).

9. Küppers, J., Surface Science Reports 22, 249-322 (1995).

10. Roth, J. and Preuss, R. and Bohmeyer, W. and Brezinsek, S. and Cambe, A. and Casarotto, E. and Doerner, E. and Gauthier, E. and Federici, G. and Higashijima, S. and Hogan, J. and Kallenbach, A. and Kirschner, A. and Kubo, H. and Layet, J.M. and Nakano, T. and Philipps, V. and Pospieszczyk, A. and Pugno, R. and Ruggiéri, R. and Schweer, B. and Sergienko, G. and Stamp, M., Nuclear Fusion 44, L21-L25 (2004).

11. Whyte, D.G. and West, W.P. and Doerner, R. and Brooks, N.H. and Isler, R.C. and Jackson, G.L. and Porter, G. and Wade, M.R. and Wong, C.P.C., Journal of Nuclear Materials 290-293, 356-361 (2001).

12. Tabarés, F.L. and Tafalla, D. and Tanarro, I. and Herrero, V.J. and Islyaikin, A. and Maffiotte, C., Plasma Physics and Controlled Fusion 44, L37-L42 (2002).

13. ITER JCT and Home Teams, Plasma Physics and Controlled Fusion 37, 19-35 (1995).

14. Drake, J.F., Physics of Fluids 30, 2429-2433 (1987).

15. Salonen, E., and Nordlund, K. and Keinonen, J. and Wu, C.H., Phys. Rev. B 63, 195415-(1-14) (2001). 